\title{
Produtividade e Eficiência Biológica de Sistemas de Recria e Engorda de Gado de Corte no Rio Grande de Sul ${ }^{1}$
}

\author{
Virginia Beretta ${ }^{2}$, José Fernando Piva Lobato ${ }^{3}$, Carlos Guilherme Mielitz Netto 4
}

\begin{abstract}
RESUMO - Foram avaliadas, por meio de simulações, a produtividade e a eficiência de sistemas de produção de gado de corte no Rio Grande do Sul, diferindo na idade de abate (IA) dos novilhos (54, 42, 36, 30, 24 e 18 meses, IA54, IA42, IA36, IA30, IA24 e IA18, respectivamente), ou nos pesos de compra e venda (sistemas especializados na recria desde 150 a $250 \mathrm{~kg}$ - R1, recria de 250 a $350 \mathrm{~kg}$ - R2 ou engorda desde 350 a $450 \mathrm{~kg}$-R3). Foi utilizado um modelo estático determinístico, representando os sistemas em equilíbrio. O manejo alimentar foi caracterizado para cada sistema com base em dados bibliográficos, conforme a IA estabelecida, enquanto os sistemas R1, R2 e R3 foram avaliados somente em campo nativo. Houve uma resposta em produtividade de tipo quadrático à redução na IA, atingindo o menor valor ( $65 \mathrm{~kg} / \mathrm{ha}$ ) no sistema IA54 e o maior no IA18 (343 kg/ha). O custo energético por quilo de peso vivo produzido aumentou em 1,19 Mcal EM/kg a cada mês a mais na IA. Associada à redução da IA, a proporção de pastagens melhoradas cresceu até $68 \%$ da área de pastejo, possibilitando um aumento paralelo da carga e do ganho médio diário. A diminuição do peso de compra e venda dos animais, para um mesmo ganho total de peso vivo, aumentou a produtividade das empresas $(\mathrm{R} 1=113$; $\mathrm{R} 2=81$; $\mathrm{R} 3=63 \mathrm{~kg} / \mathrm{ha})$, demonstrando ser a especialização na produção uma alternativa de melhoria em sistemas dependentes exclusivamente do campo nativo.
\end{abstract}

Palavras-chave: bovinos, crescimento, eficiência, produtividade, simulação, sistemas

\section{Productivity and Biological Efficiency of Growing and Finishing Beef Cattle Systems in Rio Grande do Sul}

\begin{abstract}
A static deterministic model was used to evaluate productivity and efficiency of growing and finishing beef steers systems in Rio Grande do Sul State differing at steers slaughter age (IA): 54, 42, 36, 30, 24 and 18 months (IA54, IA42, IA36, IA30, IA24 e IA18, respectively), or in market live weight for buying and sale (cattle growing systems from 150 to $250 \mathrm{~kg}-\mathrm{R} 1$, from 250 to $350 \mathrm{~kg}-\mathrm{R} 2$, or finishing cattle from 350 to $450 \mathrm{~kg}-\mathrm{R} 3$ ). Feeding management was described for each system based on published reports according to IA, while for systems R1, R2 and R3 it was considered that native pastures were the exclusive forage resource. There was a quadratic response curve between productivity and IA, with a minimum production $(65 \mathrm{~kg} / \mathrm{ha})$ for IA54 and the highest for IA18 $(343 \mathrm{~kg} / \mathrm{ha})$. Energetic cost per kg of produced live weight was linearly increased in $1.19 \mathrm{Mcal}$ ME for each month increased at IA. Reducing IA, increased the proportion of the grazing area that was occupied by improved pastures up to $66 \%$. This allowed higher stocking rates and daily gains, as well. For an equal total live weight gain, reducing steer weight for buying and sale, increased system productivity (R1: 113, R2: 81 and R3: $63 \mathrm{~kg} / \mathrm{ha}$ ), so that productive specialization appears as an alternative for improving those systems which depends exclusively on native pastures.
\end{abstract}

Key Words: cattle systems, efficiency, growing, productivity, simulation

\section{Introdução}

O aumento da produtividade da pecuária de corte no Estado de Rio Grande do Sul tem sido colocado como uma alternativa de melhoria do resultado econômico das empresas dedicadas à atividade (Pötter et al., 1998).

No caso das empresas especializadas na recria e engorda de bovinos ou de ciclo completo, elevada idade de abate dos machos reduz a taxa de desfrute e a produção de peso vivo/ hectare. Segundo dados do Anualpec (1999), no Rio Grande do Sul, os novilhos maiores de dois anos representam $36,7 \%$ dos machos em estoque, com um desfrute geral de abate de $21,1 \%$. Esta elevada idade de abate é devida ao baixo nível nutricional pós desmame, com animais mantidos basicamente em campo nativo e em altas cargas, determinando, durante o inverno, perdas de

\footnotetext{
1 Parte da tese de Doutorado em Zootecnia apresentada à Universidade Federal do Rio Grande do Sul- UFRGS, pelo primeiro autor. 2 Doutora, Departamento de Produção Animal e Pastagens, Faculdade de Agronomia, Universidade da República- Estação Experimental "Mario A. Cassinoni" Cx.P 57 072, CP: 60 000, Paysandú - Uruguai. E.mail: asimeone@adinet.com.uy

${ }^{3} \mathrm{PhD}$, Professor Adjunto IV, Dep. de Zootecnia, Fac. de Agronomia - UFRGS. Porto Alegre. Bolsista 1A do CNPq. Caixa Postal, 776 CEP: 90.0001-970 - PortoAlegre, RS. E.mail: lobato@orion.ufrgs.br

${ }^{4}$ Doutor, Professor Adjunto III do Dep.de Economia Rural, Fac. de Economia-UFRGS-Porto Alegre. RS. E.mail: mielitz@vortex.ufrgs.br
} 
peso vivo equivalentes a $25 \%$ do ganho realizado durante as estações de crescimento das pastagens (Müller \& Primo, 1986).

O Rio Grande do Sul tem farta pesquisa sobre o aumento do ganho médio diário de animais em crescimento, em diferentes estações do ano. Adequação de carga em campo nativo (Moraes et al. 1995), introdução e manejo de pastagens de espécies temperadas para o aumento da produção hibernal de forragem (Müller \& Primo, 1986; Quadros \& Maraschin, 1987; Albospino \& Lobato, 1994; Moraes \& Maraschin, 1993), de forrageiras de estação quente (Aita \& Restle, 1999; Maraschin et al. 1993), têm sido avaliadas com este objetivo. Porém, é diminuta a avaliação do impacto da incorporação do conjunto de tecnologias no sistema de produção de recria e engorda (Cezar et al., 1996; Beretta, 1999; Pötter et al., 1998, 2000).

Todavia, existem diferenças na eficiência de conversão do alimento por categorias animais, diferindo no peso vivo, na taxa de ganho e idade, associadas a variações na composição do ganho de peso vivo (Di Marco, 1993). Assim, um mesmo ganho total anual de peso vivo pode ter impactos distintos na produtividade do sistema, dependendo da categoria animal utilizada e das características da base forrageira.

Os diferentes fatores que compõem um sistema de produção interagem entre si. Uma mudança parcial, afetando apenas uma categoria animal, poderá afetar indiretamente as demais categorias do rebanho, dificultando a predição do impacto produtivo no conjunto do sistema. Assim, a avaliação do benefício de nova tecnologia deverá ser realizada considerando os seus efeitos em todo o sistema, avançando além do impacto na categoria objeto da melhoria (Black et al., 1993). A modelagem e a simulação de sistemas têm sido propostas como ferramentas de pesquisa que permitem realizar este tipo de estudo minimizando os custos e reduzindo o tempo de avaliação (Naazie et al., 1997).

A quantificação da produtividade e eficiência biológica de sistemas de engorde, variando na idade ao abate ou no peso de compra e venda dos novilhos, permitiria definir a especialização de produção de acordo com a disponibilidade de recursos naturais. Além disso, permitiria avaliar, sobre uma base objetiva, as vantagens da introdução de melhorias nos sistemas, fornecendo informações necessárias para avaliações econômicas, últimas variáveis na decisão de adoção de inovações tecnológicas.
O objetivo deste trabalho, ao usar um modelo quantitativo, foi avaliar o impacto na produtividade física e eficiência biológica de sistemas de recria e engorda, quando são introduzidas mudanças na idade de abate dos novilhos, utilizando-se pastagens melhoradas de ciclo hiberno/primaveril, bem como no peso vivo de compra e venda dos mesmos.

\section{Material e Métodos}

Foram avaliadas, mediante simulação computacional, a produtividade e a eficiência biológica de seis sistemas pecuários de recria e engorda, diferindo na idade ao abate (IA) dos novilhos: 54, 42, 36, 30, 24 e 18 meses (IA54, IA42, IA36, IA30, IA24 e IA18, respectivamente), para um mesmo peso e idade de compra. Também foram comparados três sistemas diferindo nos pesos de compra e venda: sistemas especializados na recria de novilhos de 150 a $250 \mathrm{~kg}$ (R1), de 250 a $350 \mathrm{~kg}$ (R2) ou engorda de 350 a $450 \mathrm{~kg}$ (R3).

\section{$O$ modelo}

Foi utilizado um modelo estático determinístico, representando o processo de recria e engorda nos diferentes sistemas em equilíbrio, montado com base num sistema de equações lineares simultâneas resolvidas por planilha eletrônica (Beretta, 1999). Na Figura 1 é apresentado o diagrama correspondente à estrutura do modelo de programação. O mesmo considera as principais componentes biológicas de um sistema de produção de bovinos de corte: estrutura de idades e tamanho de rebanho, características dos recursos forrageiros, manejo alimentar e sanitário das diferentes categorias ao longo do ano e superfície total da empresa. Neste trabalho, o modelo foi restrito ao manejo de categorias em crescimento e as componentes econômicas não foram consideradas.

Variações nos coeficientes da matriz de programação ou inputs do modelo, representam diferentes tecnologias utilizadas no processo de recria e engorda. Estes coeficientes foram especificados para cada um dos sistemas avaliados, utilizando como principal fonte de informação resultados experimentais gerados no Estado do Rio Grande do Sul.

\section{Coeficientes técnicos do modelo}

\section{Do sistema}

O modelo foi dimensionado para uma área determinada de empresa pecuária. A área total foi utilizada como única restrição na estimação do estoque. Foram 


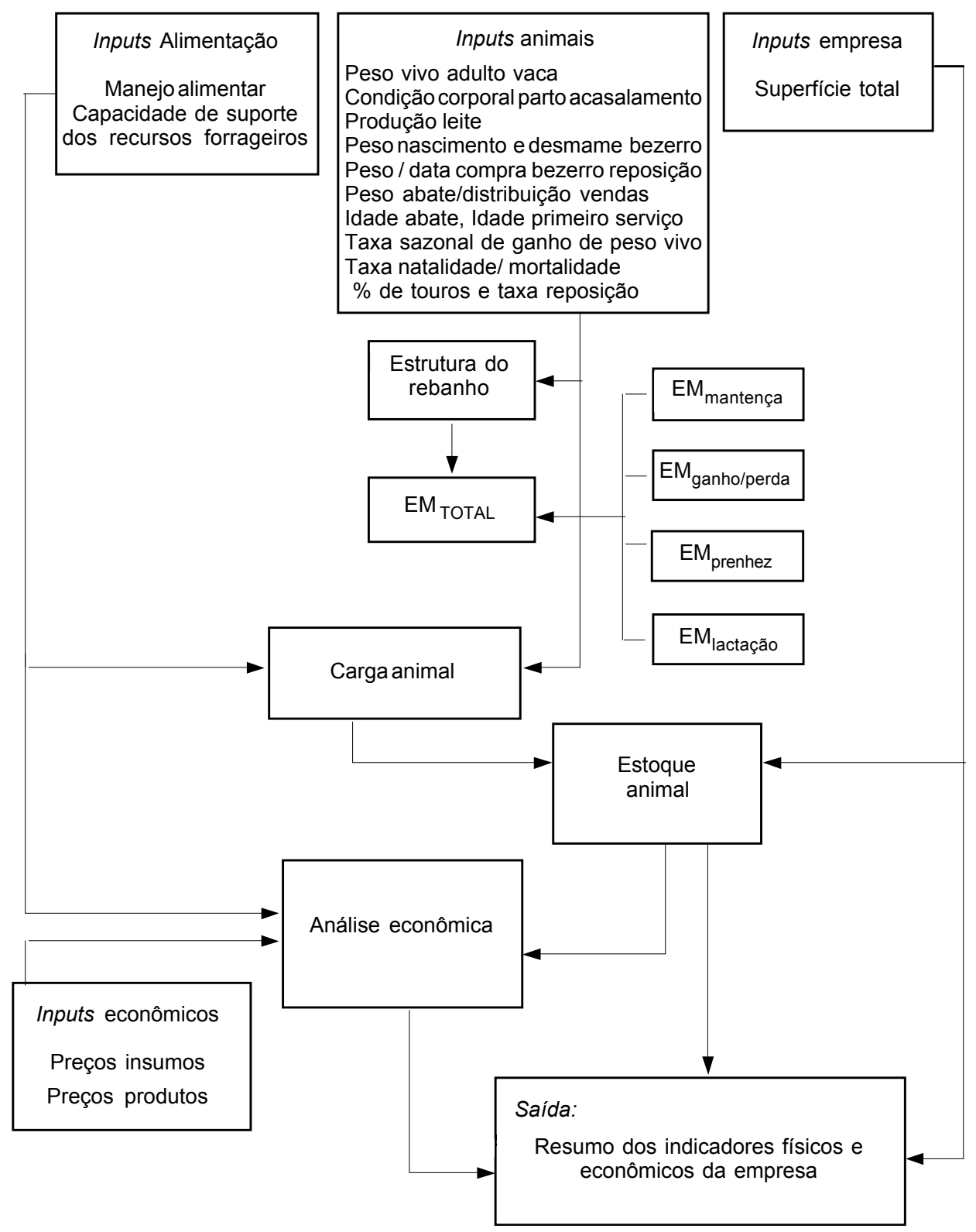

EM: energia metabolizável (metabolic energy).

Figura 1 - Estrutura interna do modelo de programação para um sistema de ciclo completo de produção de carne bovina (Beretta, 1999).

Figure 1 - Model programm structure for a beef cattle production system (Beretta, 1999).

considerados 1000 ha úteis, com o mesmo potencial produtivo dos recursos naturais para os diferentes sistemas, de forma que a produtividade não foi limitada pela variabilidade destes recursos, mas pela adoção de tecnologia, o que viabiliza as comparações. Pastagens nativas melhoradas foram consideradas como implantadas nas restevas das lavouras de verão, método comum no Rio Grande do Sul (Moraes et al., 1995), com uma vida produtiva média de três anos, antes de voltarem a ser ocupadas por lavouras.

Do rebanho

A estrutura e o tamanho do rebanho foram decorrência da IA e da taxa de mortalidade. Foi considerado que os animais de reposição entram no sistema em 
abril, com vendas distribuídas ao longo de cinco meses da safra (janeiro a maio) ou da entressafra (julho a novembro), segundo a IA considerada. $\mathrm{Na}$ avaliação do efeito da IA, todos os sistemas compraram bezerros desmamados, estruturando o rebanho em categorias de idade: seis a 12 meses; 12 a 18 meses, e assim sucessivamente, dependendo da IA. Em todos os casos foi considerado uma taxa de mortalidade de 3\% para animais de seis a 24 meses e de $1 \%$ para idades maiores.

A demanda energética para cada categoria animal do rebanho foi estimada com base nas equações do AFRC (1993), a partir da especificação das taxas de ganho sazonais por categoria, da evolução de peso vivo até o abate e do manejo alimentar. Foi considerado serem os machos castrados e da raça Hereford, vendidos para abate com um grau de acabamento constante. Devido às variações entre sistemas na velocidade de crescimento e engorde, o peso vivo de abate diferiu, sendo em média de $380 \mathrm{~kg}$ no IA18; de $440 \mathrm{~kg}$ no IA24; e de $450 \mathrm{~kg}$ nos casos restantes. A partir das exigências de energia metabolizável estimadas para cada categoria, foram calculados valores de equivalência animal tomando como base as exigências energéticas de uma unidade animal (UA) ${ }^{1}$, valor definido como unidade animal de equivalente energético (UAE). Esta base de equivalência permitiu comparar as diferentes categorias dentro de um mesmo sistema ou uma mesma categoria em diferentes sistemas de produção.

\section{Do manejo alimentar}

Variações na IA foram associadas a diferenças na taxa de ganho média anual de rebanho, que por sua vez é resultante do manejo diferenciado das várias categorias do rebanho e da incorporação de melhorias na base forrageira. As principais inovações tecnológicas consideradas, determinantes das mudanças nos coeficientes técnicos de recria e engorda, foram: i) a introdução de pastagens melhoradas de clima temperado; ii) a utilização de gramíneas anuais de verão; e iii) o ajuste da carga estacional em campo nativo. A alimentação no IA54 e IA42 foi exclusivamente em campo nativo. A redução na IA para 36 , 30 ou 24 meses foi viabilizada a partir da utilização de pastagens naturais melhoradas no período junhonovembro (Müller \& Primo, 1986; Albospino \& Lobato, 1994), variando entre sistemas as categorias manejadas nas pastagens, assim como as taxas de ganho esperadas. No IA24 as pastagens melhoradas foram utilizadas no primeiro e segundo inverno pós-desmame com taxas de ganho de 0,550 e $0,750 \mathrm{~kg} /$ animal/ dia, respectivamente. Na IA30 e IA36, as pastagens foram usadas somente no segundo inverno após o ingresso no sistema, com taxas de ganho de 0,750 e $0,700 \mathrm{~kg} / \mathrm{animal} / \mathrm{dia}$, respectivamente. Para redução da IA de 24 para 18 meses, foi considerada, além de utilização de pastagens melhoradas durante inverno/ primavera, a utilização de milheto durante o verão para os novilhos em terminação (Lupatini et al., citados por Moraes et al., 1995).

Nos sistemas R1, R2 e R3, o manejo animal em todos os casos foi feito exclusivamente em campo nativo, ingressando os animais no mês de abril e permanecendo no sistema por 12 meses, quando foram vendidos como novilho de reposição (R1 e R2) ou como novilhos gordos, para abate (R3).

A área necessária de cada um dos recursos forrageiros considerados no manejo alimentar dos diferentes sistemas foi estimada pelo modelo a partir do balanço entre o aporte energético de cada recurso por hectare e a demanda das categorias que os utilizam. O campo nativo foi caracterizado em termos de produção de matéria seca, qualidade e taxa de utilização, segundo Corrêa (1993)e Apezteguia (1994). As pastagens nativas melhoradas, misturas de Lolium multiflorum, Trifolium repens e Lotus corniculatus, foram caracterizadas segundo Quadros \& Maraschin (1987), e o milheto (Pennisetum americanum), segundo Silva et al. (1995). As pastagens permaneceram fechadas de meados de novembro a meados de janeiro, favorecendo a resemeadura das espécies exóticas introduzidas (Lobato, 1985).

Resultados e saídas do modelo

A produtividadade física do sistema foi quantificada, no modelo, como quilos de peso vivo por hectare de pastejo. A área de pastejo do sistema foi calculada como o somatório da área média anual ocupada pelos diferentes recursos forrageiros, independentemente do momento de utilização dos mesmos. Isto incluiu também o período de preparo de solo para semeadura de pastagens de verão, assim como o tempo que as pastagens melhoradas permanecem fechadas para resemeadura. A eficiência de estoque

${ }^{1} \mathrm{~A}$ unidade animal equivale a um animal de $450 \mathrm{~kg}$ de peso vivo em mantença. 
foi calculada como a relação entre os quilos produzidos e o peso vivo médio em estoque no sistema. A eficiência biológica, avaliada como a energia necessária por unidade de produto gerada no sistema, foi estimada como a relação entre a energia metabolizável (EM) consumida anualmente pelo rebanho e os quilos de peso vivo produzidos. A eficiência de conversão do alimento foi calculada como os quilos de matéria seca consumida por unidade de peso vivo ganho.

O modelo aporta também informação descritiva para cada sistema. O uso da terra, a estrutura e tamanho de rebanho, assim como a carga animal, avaliados no conjunto, permitiram interpretar as possíveis diferenças entre sistemas em produtividade e eficiência.

\section{Resultados e Discussão}

\section{Efeito da idade de abate}

Resultado das simulações, foi observado ter a redução da idade de abate, baseada na melhoria do nível alimentare da qualidade do alimento oferecido, determinado mudanças no uso do solo, na estrutura e tamanho do rebanho, as quais se refletiram na produtividade dos diferentes sistemas pecuários e na eficiência biológica do processo de recria e engorda. Na Tabela 1 são apresentados os resultados obtidos para cada sistema.

Uso da terra. A redução da IA determinou o aumento da área de pastagens no sistema de produção passando de $0 \%$ nos sistemas IA54 e IA42, baseados exclusivamente no campo nativo, para 18,5 ;

Tabela 1 - Indicadores físicos e biológicos caracterizando sistemas de recria e engorda diferindo na idade da abate dos novilhos no Rio Grande do Sul

Table 1 - Physical and biological coefficients for growing and fattening systems differing in steers slaughter age in Rio Grande do Sul

Indicadores dos sistemas de produção

Production system coefficients Idade de abate (meses) Slaughter age (months)

\begin{tabular}{|c|c|c|c|c|c|}
\hline IA & IA & IA & IA & IA & IA \\
\hline 18 & 24 & 30 & 36 & 42 & 54 \\
\hline
\end{tabular}

Uso da terra

Land use

Área de pastejo (\%) ${ }^{1}$

Grazing área

Área de lavouras $(\%)^{1}$

Crop área

Área PM $(\%)$

IP area $(\%)^{2}$

Carga média anual

Mean annual stocking rate

Animais/ha

Animals/ha

$\mathrm{UAE} / \mathrm{ha}^{3}$

AUE/ ha

GMD (g/animal/dia $)^{4}$

81,4

84,5

90,0

94,

100

100

18,6

15,5

10,0

6,0

0,0

0,0

68,0

54,9

37,5

18,5

0,0

0,0

LWG (g/animal/day)

Produtividade

Productivity

PV $(\mathrm{kg} / \mathrm{ha})^{5}$
RW
Relação IA18=100
Relation

Eficiência estoque (\%)

Stock efficiency (\%)

Eficiência biológica (Mcal EM/kg PV)

Biological efficiency (Mcal ME/ $\mathrm{kg} \mathrm{LW}$ )

$\begin{array}{llllll}1,40 & 1,21 & 1,04 & 1,00 & 0,86 & 0,90 \\ 1,32 & 1,00 & 0,93 & 0,84 & 0,72 & 0,74 \\ 671 & 541 & 410 & 324 & 268 & 198\end{array}$

1 Área expressa como porcentagem da supefície total da empresa (Area expressed as percentage of systems total area).

2 PM: pastagens melhoradas, área expressa como porcentagem da área de pastejo (IP: improved pastures, area expressed as percentage of grazing area).

3 UAE: unidade animal de equivalente energia. Uma UAE equivale às exigências diárias de energia metabolizável de um animal de $450 \mathrm{~kg}$ de peso vivo em mantença (AUE: animal unit energy equivalent. One AUE = daily requirements of metabolic energy peranimal of $450 \mathrm{~kg}$ in maintenance).

4,5 GMD: ganho médio diário; PV: peso vivo (LWG: live weight gain; LW: live weight). 
37,5; 54,9; e 68,0\% nos sistemas IA36, IA 30, IA24 e IA18, respectivamente. Sem considerar o IA18, que, além das pastagens nativas melhoradas, utilizou também milheto durante o verão, a porcentagem de pastagens melhoradas aumentou em 17,2\% para cada seis meses de redução da IA ( $\mathrm{y}=121,98-2,8733 \mathrm{x}$; $\left.\mathrm{R}^{2}=0,99\right)$. Paralelamente, foi registrada diminuição da área de pastejo devido a integração da lavoura de verão em sistemas com utilização de pastagens, uma vez que estas foram semeadas na resteva. Embora este fato não afete diretamente a produtividade por unidade de área destinada à produção animal, pode incidir sobre a margem bruta pecuária e o resultado econômico global da empresa. Simeone (1999) reporta para sistemas comerciais de engorda intensiva no Uruguai, também com integração lavoura-pecuária, $55 \%$ da área de pastejo ocupada por pastagens melhoradas.

Estrutura de estoque e tamanho do rebanho. A estrutura de estoque exerceu marcado efeito na produtividade e eficiência biológica. Num sistema de recria e engorda em equilíbrio, a estrutura de idades do rebanho evidencia a duração do processo produtivo, determinando o número de novilhos mantidos no sistema para cada 100 animais comprados anualmente. Para uma área de empresa dada, isto afeta o total de animais que possam ser comprados a cada ano e, consequentemente, a produção total e taxa de abate.

Os resultados das simulações mostraram que o número de animais em estoque para cada 100 bezerros comprados anualmente diminuiu na medida que os sistemas de produção se intensificaram, associado à diminuição no número de categorias de novilhos de diferentes idades (Figura 2). Enquanto no IA54 existiam em estoque 374 animais para cada 100 bezerros comprados, no IA24 havia apenas 138 animais, caindo este valor para 90 animais no IA18. A mesma tendência registrou-se na área ocupada por cada um destes rebanhos: 421 e 64 ha a cada 100 bezerros entrados nos sistemas IA54 e IA18, respectivamente (Figura 2). A redução do tamanho do rebanho conforme diminuiu a idade ao abate foi linear, caindo o estoque em 47,3 cabeças/ 100 bezerros comprados para cada seis meses de redução na IA. Já a magnitude do impacto na área dependeu da mudança qualitativa nos recursos forrageiros do sistema. Diminuir em 12 meses a IA, passando de 54 para 42 meses, determinou uma redução de $25,1 \%$ no estoque e de $19,7 \%$ na área que este ocupa. Esse menor impacto na área

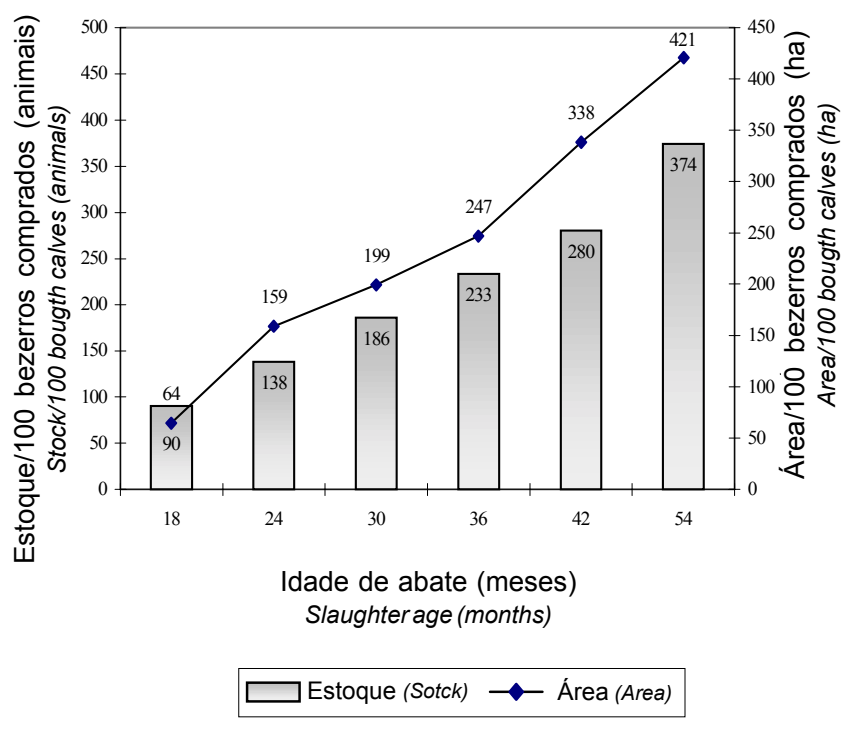

Figura 2 - Área em hectare e estoque animal/ 100 bezerros comprados anualmente em sistemas de recria e engorda no Rio Grande do Sul, diferindo na idade ao abate dos novilhos: $18,24,30,36$, 42 ou 54 meses.

Figure 2 - Area and animal stock/100 calves bought in growing and fattening systems in Rio Grande do Sul, differing in steers slaughter age: $18,24,30,36,42$ or 54 months.

do que no estoque é explicado pelo fato de não ter havido alteração na base forrageira, que continuou sendo somente campo nativo. A redução da IA foi feita com aumento na taxa de ganho a expensas de redução da carga animal. Já uma mudança na IA da mesma magnitude, mas passando de 42 para 30 meses, equivaleu à redução de $33,5 \%$ no estoque e de $41,1 \%$ na área ocupada por este. Esta mudança nos coeficientes técnicos do sistema foi feita na base da incorporação de pastagens nativas melhoradas, utilizadas durante o inverno-primavera pelos novilhos de 18 a 24 meses de idade. Os campos nativos liberados pelas categorias de novilhos de 30 a 36 meses e de 36 a 42 meses, já não existentes no sistema IA30, passaram a ser ocupados por animais de recria, mais novos e de menor peso vivo, passando os animais em terminação a ser manejados numa área menor. $\mathrm{Na}$ medida em que as pastagens melhoradas foram incorporadas ao sistema, houve aumento na capacidade de carga da base forrageira. Em termos gerais, quanto maior a incorporação de pastagens destinadas a diminuir a IA, maior foi a redução percentual na área necessária para cada 100 bezerros comprados. 


\section{Produtividade e eficiência de estoque}

A produtividade dos sistemas de recria e engorda, avaliada como os quilos de peso vivo produzidos/ ha de pastejo, aumentou conforme foi diminuída a IA (Tabela 1). Porém, observou-se que, para uma variação na IA da mesma magnitude, o impacto produtivo foi maior quanto menor foi a idade em que operou-se a mudança. Diminuir em 12 meses a IA representou aumento de $29,2 \%, 84,5 \%, 100 \%$ ou $121,2 \%$, conforme este foi implementado em sistemas abatendo seu novilhos com 54, 42, 36 ou 30 meses, respectivamente. Este tipo de resposta foi explicado pela mudança conjunta na carga animal e na taxa média diária de ganho de peso vivo, ambas dependentes das características da base forrageira em cada sistema. À medida que a IA diminuiu, foram necessárias maiores taxas de ganho de peso. A resposta em carga foi variável dependendo das mudanças introduzidas na base forrageira. A substituição do campo nativo por pastagens nativas melhoradas, além de viabilizar maiores taxas de ganho, permitiu aumentar a carga média do sistema. Foi o observado ao comparar os sistemas IA36, IA30 e IA24, onde a superfície de pastagens nativas melhoradas cresceu quanto menor foi a IA, aumentando a carga em $21 \%$. Porém, quando a IA foi reduzida sem modificar-se a base forrageira, caso da comparação dos sistemas IA54 e IA42, registrou-se aumento da taxa de ganho de peso vivo/ ha de $29,2 \%$ as expensas da redução da carga animal.

$\mathrm{O}$ aumento da carga, na medida em que os sistemas se intensificaram, foi feito a partir da melhoria da capacidade de suporte da base forrageira, acontecendo uma mudança do que seria a ótima pressão de pastejo ao passar de um sistema para outro. Aumentos de carga que não considerarem modificações na base forrageira, provocarão uma redução na produtividade uma vez que o acréscimo no número de animais por unidade de área não compensa o pior desempenho individual (Hodgson, 1990). De fato, diversos autores atribuem a baixa produtividade de sistemas pecuários tradicionais à superlotação atual dos campos nativos do Rio Grande do Sul, em relação à capacidade de suporte real (Jacques, 1999). O ajuste estacional da carga é uma alternativa para o aumento da produtividade. Potenciais de produção de peso vivo a partir do campo nativo da região da Depressão Central do Rio Grande do Sul, da ordem de $125 \mathrm{~kg} /$ ha com ofertas de forragem de $12 \%$ do peso vivo durante a estação de crescimento (período setembro-maio), são reportados pela pesquisa (Maraschin \& Jacques, 1993). Esse valor dobra a produção predita para o sistema IA54 e se corresponde, quando considerada numa base anual, com os $87 \mathrm{~kg} /$ ha/ano obtidos por simulação para o sistema IA42, sustentado exclusivamente sobre pastagens nativas. Isto corrobora com o potencial de melhoria dos indicadores da pecuária a partir do ajuste estacional da carga.

A capacidade de suporte hibernal do campo nativo foi o fator limitante do aumento da produtividade em sistemas que dependem exclusivamente deste recurso. Na medida que esta limitante foi levantada a partir da introdução de espécies exóticas de ciclo hibernal/primaveril, registrou-se um aumento no nível de produção de peso vivo por hectare de pastejo. Os resultados das simulações mostram uma relação linear positiva entre estas variáveis, quando avaliadas em sistemas cuja base forrageira são pastagens nativas e melhoradas $(\mathrm{PV}[\mathrm{kg} / \mathrm{ha}]=74,159+2,699$ [\%pastagens melhoradas], $\left.\mathrm{R}^{2}=0,94\right)$. A utilização de pastagens nos meses de junho/julho a outubro/novembro, possibilitou manejar uma maior carga animal durante o inverno, quando a produção de forragem do campo nativo é mínima, e potencializou a utilização da pastagem nativa durante a estação quente, quando apresenta a maior produtividade. Esta complementação dos diferentes recursos forrageiros do sistema aumentou a eficiência global de utilização da forragem, explicando o aumento da carga média anual e a maior produtividade de sistemas mais intensivos. No Uruguai, Simeone (1999) reporta para sistemas comerciais pastoris especializados na engorda intensiva de bovinos, cargas médias de $464 \mathrm{~kg}$ peso vivo/ha, com $66 \%$ da área de pastejo de nativas melhoradas com azevém e trevo branco e uma produção de peso vivo de $341 \mathrm{~kg} / \mathrm{ha}$. A carga destes sistemas invernadores representa um aumento de mais $50 \%$ sobre a carga média utilizada nos sistemas baseados somente em campo nativo. Estes resultados estão em concordância com os previstos pelos modelos de simulação ao comparar os sistemas IA54 e IA18.

\section{Eficiência biológica}

A eficiência de produção melhorou na medida que os processos se intensificaram. A energia consumida por quilo de peso vivo apresentou uma relação positiva e direta com a IA, aumentando em $1,19 \mathrm{Mcal} \mathrm{EM} / \mathrm{kg}$ para cada mês a mais na IA. Isto representou ser a energia necessária por quilo produ- 
zido no sistema IA52 o triplo do necessário no IA18 (60,7 vs 20,2 Mcal EM/ kg). Estas diferenças foram explicadas, basicamente, pela redução percentual dos custos de mantença em relação à EM total consumida anualmente pelo rebanho ao ser reduzida a IA (Figura 3). A energia destinada para mantença é a componente mais importante das exigências animais (Ferrell \& Jenkins, 1984), de forma que, mudanças nos coeficientes técnicos que reduzem a proporção do total consumido destinado a este processo, reduzem o custo energético de produção. A menor duração do ciclo produtivo e a maior taxa de ganho média anual quanto menor for a IA, reduzem a proporção relativa de energia consumida destinada a mantença. Por outra parte, a melhoria na qualidade da base forrageira, fundamentalmente a incorporação de pastagens nativas melhoradas e gramíneas anuais de verão, determinou aumento na eficiência de uso da energia consumida para os processos de deposição de tecidos $\left(\mathrm{k}_{\mathrm{g}}\right)$ (AFRC, 1993). De acordo com a relação colocada por estes autores, a deposição

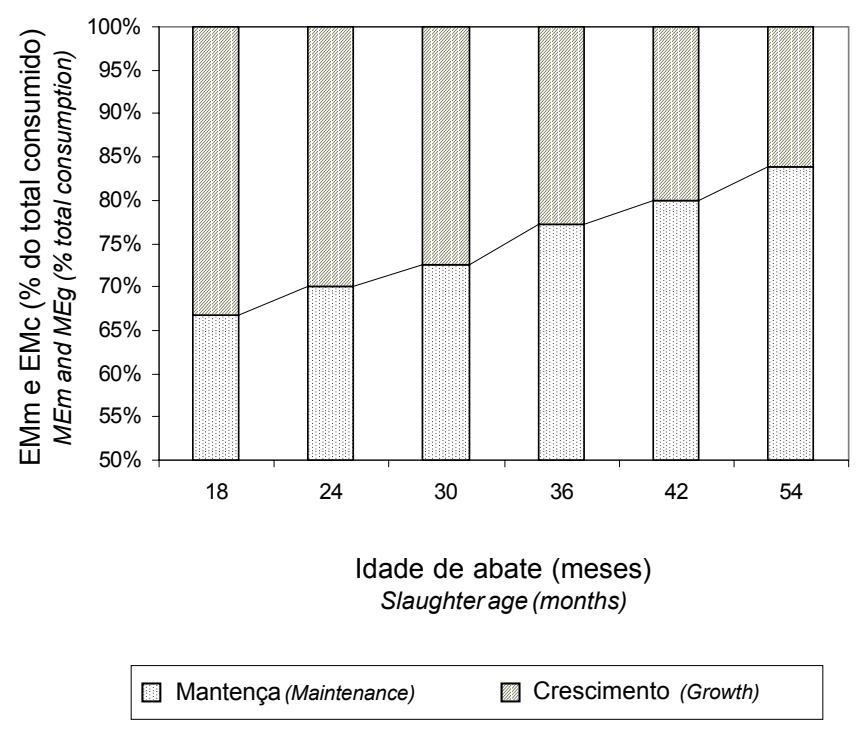

Figura 3 - Partição da energia metabolizável consumida (EM) para as funções de mantença (EMm) e crescimento (EMc) em sistemas de recria e engorda no Rio Grande do Sul, diferindo na idade de abate dos novilhos: 18, 24, 30, 36, 42 ou 54 meses.

Figure 3 - Partition of consumed metabolic energy for maintenance (MEm) and growth (MEg) in growing and fattening systems in Rio Grande do Sul differing in steers slaughter age: $18,24,30,36,42$ or 54 months. de tecidos passou de $0,37 \mathrm{~kg}$ para $0,43 \mathrm{~kg}$ quando os novilhos utilizaram o campo nativo ou as pastagens nativas melhoradas durante o inverno, respectivamente, reduzindo as exigências de EM e os quilos de matéria seca consumida por quilo produzido.

Cundiff et al. (1981, 1984), Dikeman et al (1985), Lamb et al. (1992) e Williams et al. (1995) reportaram valores de eficiência de conversão para a fase de crescimento e engorda variando entre 16,4 e $29,8 \mathrm{Mcal} \mathrm{EM} / \mathrm{kg}$ de peso vivo produzido, dependendo da taxa de crescimento, do genótipo e do critério de abate (peso, idade ou grau de acabamento). A maioria destes trabalhos corresponde a sistemas de alimentação em confinamento, com alta concentração energética e com a IA não superando os 24 meses, o que explica a semelhança com as eficiências registradas nos sistemas IA18 e IA24.

De acordo com as diferenças registradas entre as cinco estratégias de engorda representadas nos modelos estudados, Williams et al. (1995), avaliando o efeito do sistema de recria na eficiência de engorda, por intermédio de um modelo dinâmico, observaram que menores taxas de ganho e períodos de recria prolongados, resultaram em animais mais velhos ao abate, maiores exigências de mantença para realizar o mesmo ganho total de peso vivo e pior eficiência biológica. Os mesmos autores colocam que, quando os novilhos são recriados durante longos períodos de tempo com taxas de ganho de peso muito baixas, a alternativa posterior de serem colocados em dietas de acabamento (confinamento) poderia melhorar a eficiência biológica global, uma vez que o peso de carcaça aumenta.

Efeito do peso vivo de compra e venda dos novilhos

$\mathrm{Na}$ Tabela 2 estão apresentados os resultados das simulações para os sistemas R1, R2 e R3. Estes sistemas diferenciam-se no peso vivo médio de compra e venda dos novilhos. Porém, o peso vivo total ganho/ animal foi o mesmo nos três casos. Observou-se que, quanto maior foi o peso vivo de entrada e saída dos novilhos, menor foi a produção de peso vivo/ ha, registrando-se redução de $28 \%$, ao passar do R1 para o R2 e de $22 \%$, ao passar do R2 para o R3. Ao avaliar as duas componentes da produtividade, carga e ganho individual, verificou-se que, para uma taxa de ganho variando entre 0,270 a $0,285 \mathrm{~kg} / \mathrm{animal} / \mathrm{dia}$, o sistema R1, trabalhando com animais menores, permitiu manter maiores cargas médias quanto menor foi o peso de compra ( 1,$08 ; 0,8$ e 0,64 cabeças/ ha, R1, R2 e R3, respectivamente), traduzindo-se em maior 
Tabela 2 - Indicadores físicos e biológicos caracterizando sistemas especializados na recria ou engorda no Rio Grande do Sul, diferindo no peso vivo de compra e venda dos novilhos: 150 a $250 \mathrm{~kg}$ (R1), 250 a $350 \mathrm{~kg}$ (R2) ou 350 a $450 \mathrm{~kg}\left(\mathrm{R}^{3}\right)$

Table 2 - Physical and biological coefficients for growing or fattening systems in Rio Grande do Sul, differing in calf/l steer live weight for buying and sale: 150 to $250 \mathrm{~kg}(R 1), 250$ to $350 \mathrm{~kg}(R 2)$ or 350 to $450 \mathrm{~kg}(R 3)$

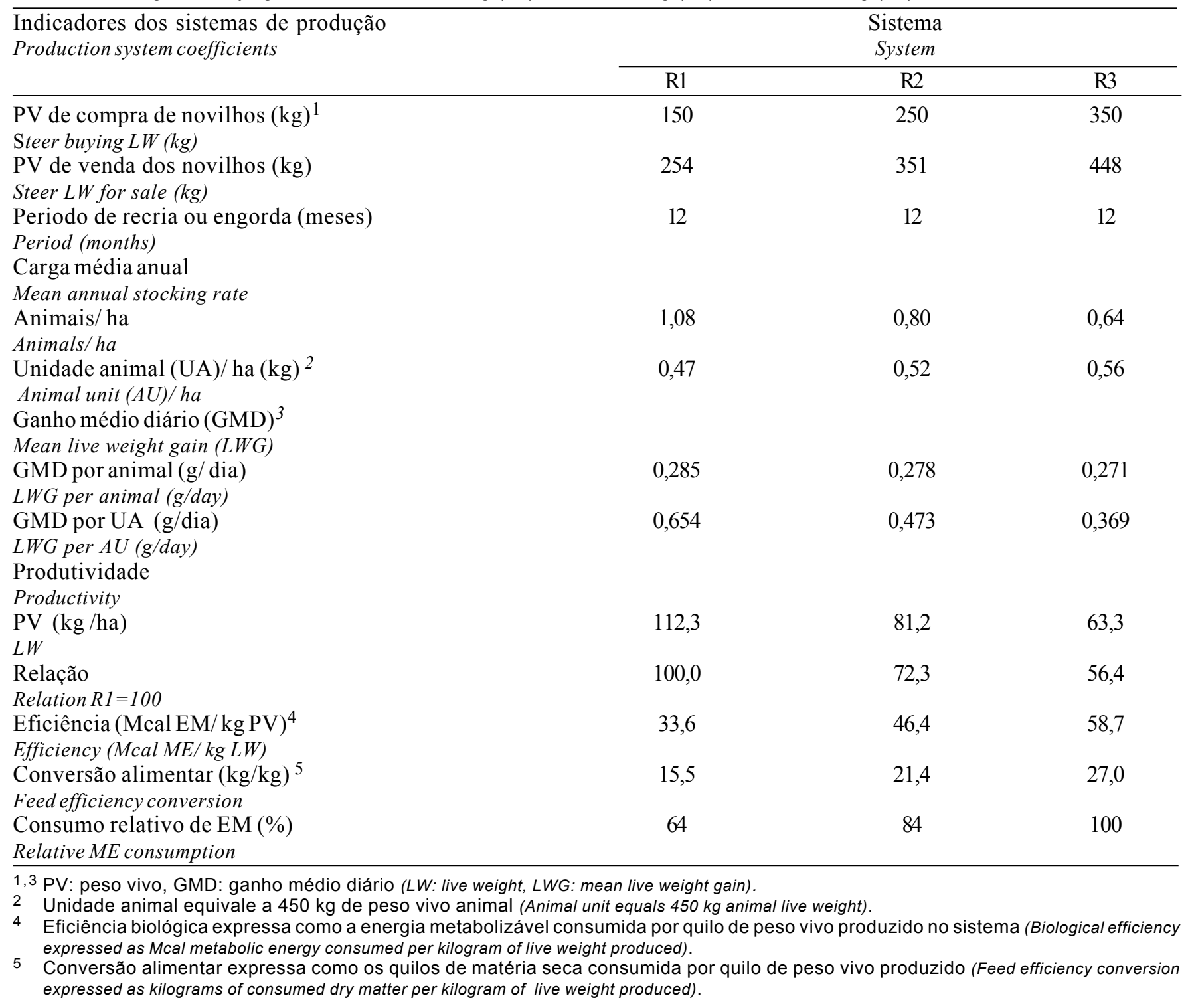

produção de peso vivo/ha. Do ponto de vista do balanço energético entre as exigências animais e o aporte do campo nativo para uma mesma capacidade de suporte, a taxa de ganho para cada $450 \mathrm{~kg}$ de peso vivo mantidos no campo aumentou quanto menor foi o peso vivo de compra do gado $(\mathrm{R} 1=0,654 ; \mathrm{R} 2=0,473 ; \mathrm{R} 3=0,369 \mathrm{~kg} / \mathrm{UA} / \mathrm{dia}) . \mathrm{O}$ peso vivo médio do rebanho aumentou quanto maior foi o peso de compra-venda. Assim, enquanto no sistema R3 para ganhar 0,271 kg/animal/dia foi preciso manter $396 \mathrm{~kg}$ de peso vivo médio, no sistema R2 este valor diminuiu para $296 \mathrm{~kg}$ e no R1, para $196 \mathrm{~kg}$, fazendo com que o mesmo ganho de peso seja feito a partir de um menor custo fixo de mantença. Estas diferenças se refletiram na eficiência de estoque, cujos valores foram 57,$9 ; 36,1$; e $25,9 \%$ para o R1, R2 e R3, respectivamente.

O custo energético por quilo de peso vivo aumentou na medida que o peso vivo médio do rebanho aumentou. Isto foi associado a um aumento nas exigências de EM/ animal, tanto para mantença quanto para produção. A eficiência biológica é 
máxima imediamente após o desmame, diminuindo na medida em que avança o período pós-desmame, como resultado de um aumento nas exigências totais de mantença, de uma diminuição na taxa de ganho e de um aumento na proporção desse ganho, correspondente ao acúmulo de gordura (Di Marco, 1993). Embora nestes sistemas a taxa média de ganho diário seja similar, a composição do ganho muda, aumentando a proporção de gordura em relação ao músculo, quanto maior for o peso vivo do animal e, conseqüentemente, o custo energético por quilo de peso vivo depositado.

A avaliação global dos resultados de simulações de sistemas de recria e engorda de bovinos de corte no Rio Grande do Sul mostrou que a diminuição do peso de compra e venda dos animais, para um mesmo ganho total de peso vivo, passando de $350 \mathrm{~kg}$ para $150 \mathrm{~kg}$, é uma alternativa que equipara o impacto produtivo registrado pela introdução de até $18,5 \%$ de pastagens e a redução da IA de 54 para 36 meses, quando é feito todo o processo de recria e engorda na mesma empresa (um aumento do peso vivo/ ha de $77,4 \%$ vs $83,1 \%$, respectivamente). Assim, existiu correspondência entre a IA que minimiza o custo energético por quilo de peso vivo produzido e a que maximiza a produção de peso vivo/ha. Porém, coloca-se a questão de qual a IA que maximiza o resultado econômico. As condições de aptidão agroecológica para introdução de pastagens, assim como as conjunturas de mercados, as relações de preços entre o quilo do novilho magro para reposição e o quilo do novilho gordo, poderão condicionar a escolha da melhor alternativa produtiva.

O impacto da introdução de pastagens hiberno/primaveris, destinadas ao manejo de uma determinada categoria, na redistribuição da área de pastejo entre as restantes categorias do rebanho, destaca a importância da avaliação da viabilidade de uma nova técnica, considerando o funcionamento global da empresa e não apenas as categorias diretamente envolvidas. Por sua vez, a avaliação em nível de sistema de produção dilui no ano a produção/ha em pastagens melhoradas durante o período de uso efetivo das mesmas ( 6 a 7 meses), o que explica também, em parte, o menor impacto produtivo registrado a partir das simulações em relação aos valores obtidos em níveis experimentais.

\section{Conclusões}

Em sistemas de recria e engorda de gado de corte, a produção de peso vivo/hectare de pastejo aumentou e o custo energético por quilo de peso produzido diminuiu, conforme foi reduzida a idade de abate dos novilhos. Esta melhoria, baseada na incorporação de tecnologia provada em nível experimental, como o ajuste da carga em campo nativo, a melhoria do campo nativo através da introdução de espécies de ciclo hiberno/primaverile, por último, a utilização de gramíneas anuais de verão, permitiu aumentar gradualmente a produtividade dos sistemas pecuários do Rio Grande do Sul.

A especialização em determinada fase do processo de recria e engorda, como a recria de animais jovens a serem vendidos para sistemas com pastagens melhoradas ou para terminação em confinamento, foi uma alternativa para a melhoria do resultado produtivo, quando não foi modificada substancialmente a base forrageira.

A resposta global positiva do sistema a melhorias da base forrageira, validou, do ponto de vista biológico, a tendência de respostas obtidas em nível de áreas experimentais. Porém, a magnitude da resposta pode mudar, o que confirma a necessidade da avaliação do impacto global, que apresenta uma nova técnica, antes de sua adoção pelo produtor.

\section{Literatura Citada}

AITA, V.; RESTLE, J. Produção animal em pastagens de estação quente. In: REUNIÃO ANUAL DA SOCIEDADE BRASILEIRA DE ZOOTECNIA, 36., 1999, Porto Alegre. Anais... Porto Alegre: Sociedade Brasileira de Zootecnia, 1999. p.13.

AGRICULTURAL RESEARCH COUNCIL - AFRC. Energy and protein requirements of ruminants. An advisory manual prepared by the AFRC Technical Committee on Responses to Nutrients. Wallingford: CAB International, 1993. 159p.

ALBOSPINO, B.H.; LOBATO, J.F.P. Efeitos do desmame precoce de bezerros no desempenho até os 24-26 meses de idade. Revista Brasileira de Zootecnia, v.23, n.4, p.565-575, 1994.

ANUALPEC: Anuário da pecuária brasileira. São Paulo: Argos Comunicação, 1999. 447p.

APEZTEGUÍA, E. Potencial produtivo de uma pastagem natural do Rio Grande do Sul, submetida a distintas ofertas de forragem. Porto Alegre: Universidade Federal do Rio Grande do Sul, 1994. 169p. Dissertação (Mestrado em Agronomia) - Universidade Federal do Rio Grande do Sul, 1994.

BERETTA, V. Avaliação bioeconômica de sistemas alternativos de produção de gado de corte no Rio Grande do Sul. Porto Alegre: Universidade Federal do Rio Grande do Sul, 1999. 204p. Tese (Doutorado em Zootecnia) - Universidade Federal do Rio Grande do Sul, 1999. 
BLACK, J.L.; DAVIES, G.T.; FEMING, F.F. Rol of computer simulation in the applications of knowledge to animal industries. Australian Journal Agriculture Research, v.44, n.3, p.541-555, 1993.

CEZAR, I.M.; EUCLIDES FILHO, K; MARTINS, C.I. Novilho precoce: reflexos na eficiência e economicidade do sistema de produção. Campo Grande: EMBRAPA/CNPGC, 1996. 31p. (Documentos, 66). (Resumo na base de dados CAB, Wallingford, CD-ROM 1996-1998)

CORREAA, F.L. Produção e qualidade de uma pastagem nativa do Rio Grande do Sul sob níveis de oferta de forragem à novilhos. Porto Alegre: Universidade Federal do Rio Grande do Sul, 1993. 165p. Dissertação (Mestrado em Agronomia) - Universidade Federal do Rio Grande do Sul, 1993.

CUNDIFF, L.V.; KOCH, R.M.; GREGORY, K.E. et al. Characterization of biological types of cattle - Cycle III. IV. Post weaning growth and feed efficiency of steers. Journal of Dairy Science, v.53, n.2, p.332-346, 1981.

CUNDIFF, L.V.; KOCH, R.M.; GREGORY, K.E. Characterization of biological types of cattle (Cycle III). IV. Post weaning growth and feed efficiency. Journal of Animal Science, v.58, n.2, p.312-323, 1984.

DI MARCO, O.N.. Crecimiento y respuesta snimal. Mar del Plata: Asociación Argentina de Producción Animal, 1993. $129 \mathrm{p}$.

DIKEMAN, M.; NAGELE, K.; MYERS, S. et al. Accelerated versus conventional beef production and processing. Journal of Animal Science, v.61, n.1, p.137, 1985.

FERRELL, C.L.; JENKINS, T.G. Energy utilization by mature, nonpregnant, nonlactating cows of different types. Journal of Animal Science, v.58, n.1, p.234-243, 1984.

HODGSON, J. Grazing management: science into practice. New York: Longman Scientific \& Technical, 1990. 203p.

JACQUES, A.V.A. Potencialidade das pastagens do Rio Grande do Sul visando à intensificação da pecuária. In: LOBATO, J.F.P.; BARCELLOS, J.; KESSLER, A.M. (Eds.) Produção de bovinos de corte. Porto Alegre: EDIPUCRS, 1999. p.67-76.

LAMB, M.A.; TESS, M.W.; ROBINSON, O.W. Evaluation of mating systems involving five breeds for integrated beef production systems: II. Feedlot segment. Journal of Animal Science, v.70, n.3, p.700-713, 1992.

LOBATO, J.F.P. Gado de cria. Tópicos. Porto Alegre: Adubos Trevo, 1985. 32p.

MARASCHIN, G.E., JACQUES, A.V.A. Grassland opportunities in the supbtopical region of South America In: INTERNATIONAL GRASSLAND CONGRESS, 17., 1993, Palmerston North. Proceedings... Palmerston North, New Zealand: New Zealand Grassland Association, 1993.p.19771981.

MARASCHIN, G.E.; MORAES, A.; SILVA, L.F.A. et al. Cultivated pasture, forage on offer and animal response. In: INTERNATIONAL GRASSLAND CONGRESS, 17., 1993, Palmerston North. Proceedings... Palmerston North: New Zealand Grassland Association. 1993. p.2014-2015.
MORAES, A.; MARASCHIN, G.E. Animal production and dynamics of pangola grass, rye grass and white clover pasture as influenced by grazing pressure. In: INTERNATIONAL GRASSLAND CONGRESS, 17., 1993, Palmerston North. Proceedings... Palmerston North: New Zealand Grassland Association, 1993. p.2015-2016.

MORAES, A.; MARASCHIN, G.E.; NABINGER, C. Pastagens nos ecossistemas de clima subtropical: pesquisas para o desenvolvimento sustentável. In:ANDRADE, R.P., BARCELLOS, A., ROCHA, C.M. (Ed). SIMPÓSIO SOBRE PASTAGENS NOS ECOSSISTEMAS BRASILEIROS, 1995, Brasília. Simpósio... Brasília: Sociedade Brasileira de Zootecnia, 1995. p.147-200.

MÜLLER, L.; PRIMO, A.T. Influência do regime alimentar no crescimento e terminação de bovinos e na qualidade da carcaça. Pesquisa Agropecuária Brasileira, v.21, n.4, p.445-452, 1986.

NAAZIE, A.; MAKARECHIAN, M.; HUDSON, R.J. Efficiency of beef production systems: description and preliminary evaluation of a model. Agriculture System (Resumo em base CAB, Wallingford, CD-ROM 1996-1998). v.54, n.3, p.357-380, 1997.

PÖTTER, L.; LOBATO, J.F.P.; MIELITZ NETTO, C.G.A. Produtividade de um modelo de produção para novilhas de corte primíparas aos dois, três e quatro anos de idade. Revista Brasileira de Zootecnia, v.27, n.3, p.613-619, 1998.

PÖTTER, L.; LOBATO, J.F.P.; MIELITZ NETTO, C.G.A. Análises econômicas de modelos de produção com novilhas de corte primíparas aos dois, três e quatro anos de idade. Revista Brasileira de Zootecnia, v.29, n.3, p.861-870, 2000.

QUADROS, F.L.P.; MARASCHIN, G.E. Desempenho animal em misturas de espécies forrageiras de estação fria. Pesquisa Agropecuária Brasileira, v.22, n.5, p.535-541, 1987.

SILVA, A.W.; MACEDO, A.F.; FRANCISCATO, C. Produção de matéria seca de milheto, sorgo sudão e teossinto, sob diferentes épocas de semeadura no Planalto Serrano Catarinense. In: REUNIÃO ANUAL DA SOCIEDADE BRASILEIRA DE ZOOTECNIA, 32., 1995, Brasília. Anais... Brasília: Sociedade Brasileira de Zootecnia, 1995. p.13.

SIMEONE, A. Producción intensiva de carne. Revista de Fucrea, n.205, p.16-20, 1999.

WILLIAMS, C.B.; BENNETT, G.L.; KEELE, J.W. Simulated influence of post weaning production system on performance of different biological types of cattle: III. Biological efficiency. Journal of Animal Science, v.73, n.3, p.686-698, 1995.

Recebido em: 20/06/01 Aceito em: 12/11/01 